\title{
Do Patient/Doctor Benefits from Telephone/Electronic Contacts Exceed Those of Face-To-Face Visits?
}

\author{
Josef Finsterer ${ }^{a}$ Fulvio A. Scorza ${ }^{b}$ \\ aKlinik Landstrasse, Messerli Institute, Vienna, Austria; ${ }^{\text {bD }}$ isciplina de Neurociência, Universidade Federal de São \\ Paulo/Escola Paulista de Medicina (UNIFESP/EPM), São Paulo, Brazil
}

\section{Dear Editor,}

We appreciate the reply by Wenninger et al. [1] to our letter to the editor [2] and for providing additional valuable information. The specification of their procedures helps understand the methods they applied [1]. We comprehend that Wenninger et al. [1] defend their procedures; however, there are still some issues that require discussion.

Symptoms are only one aspect of a disease. Though symptoms can be transmitted via telephone or electronic media, they may not accurately reflect the current status of a disease. Since myotonic dystrophy is a progressive disease, progression and eventual consequences have to be objectively evaluated. It is the clinician who has to assess whether, for example, muscle weakness and wasting, cognitive function, or heart function progressed since the last control and has to validate the patient's risk of experiencing putative complications and what tentative actions have to be taken. Collecting only symptoms in a telephone call may miss signs that the patient does not recognize.

Concerning the burden of travelling, many patients like to travel despite being physically or mentally handicapped. For some of them, it is even an adventure and they look forward to see the doctor again, particularly when the relation had lasted for longer already. Travelling

karger@karger.com

(c) 2021 S. Karger AG, Basel

www.karger.com/ene

Karger! may provide the feeling of being integrated and accepted by the society. Being in the public gives these patients the chance to socialize. Of course, this may depend on the individual character and physical condition, but for most patients, the advantages of personal contacts outweigh the disadvantages of travelling. Telephone interviews may contribute to the isolation of a handicapped person.

We do not agree with the notion that the number of face-to-face visits can be reduced by online interviews. On the contrary, when the patient is not physically present, the electronic patient/doctor interactions may have to be intensified instead to compensate for missing on-site contacts. Only if the patient is truly unwilling or physically unable to attend the doctor, it is better to carry out a telephone interview than to have no information at all. Also for study purposes, it may be acceptable to collect data about symptoms online. Disregarding these exceptions, the personal contact should be favoured over the electronic contact, as the face-to-face contact provides more information about the physical condition and degree of progression since the last contact and the patient's interaction with the society and the health care system than distant ear-to-ear interactions. For routine follow-ups, the personal contact and the face-to-face interaction should not be given up. Telephone interviews may suggest that the doctor has no time or no interest in the patient. 
There is, of course, a general tendency of standardizing instead of individualizing medicine. Checklists, standards of procedures, questionnaires, scores, and assessment tools are increasingly applied, but it remains questionable if the individual gets lost within this automation of medical procedures. Of course we get a quick overview when we know, for example, the Hunt-Hess score, the Glasgow Coma score, or the NIHSS, but applying these tools favours abstracting the individual and reducing it to figures, which may be misleading with regard to individual survival or intensive measures to be taken. The need of individuals acting on either side of the doctor/patient interactions to be respected still remains.

\section{Statement of Ethics}

Not applicable.

\section{Conflict of Interest Statement}

There is no conflict of interest to declare.

\section{Funding Sources}

The work received no funding.

\section{Author Contribution}

J.F. contributed to the design, literature search, discussion, first draft, and critical comments. F.S. contributed to the literature search, discussion, critical and comments and gave the final approval.

\section{Data Availability Statement}

All data reported are available from the corresponding author.
References
1 Wenninger S, Stahl K, Montagnese F, Schoser B. Utility and results from a patient-reported online survey in myotonic dystrophies types 1 and 2. Eur Neurol. 2020;83(5):523-33.

2 Finsterer J, Scorza FA. Do not replace personal patient/doctor interactions by electronic visits in myotonic dystrophy patients. Eur Neurol. 2021. https://doi.org/10.1159/000516773. 